\title{
Pseudoangiomatous Stromal Hyperplasia
}

National Cancer Institute

\section{Source}

National Cancer Institute. Pseudoangiomatous Stromal Hyperplasia. NCI Thesaurus.

Code C104373.

A benign proliferation of the stromal cells in the breast. It is classified as simple, when associated with the presence of slit-like spaces without erythrocytes and as fascicular/proliferative, when spindle cell proliferation without atypia is present. It is often seen in breast tissue specimens as small foci associated with benign epithelial lesions. Pseudoangiomatous stromal hyperplasia presenting as a well-circumscribed palpable mass is rare. 